\title{
Migration und Bildungschancen
}

\section{Erfahrungen aus einem bundesweiten Projekt der Bildungsberatung für Familien}

\author{
DJAMILA YOUNIS \\ Djamila Younis ist Referentin für Bil- \\ dungspartnerschaften unter Betei- \\ ligung von Migrantenfamilien beim \\ Deutschen Roten Kreuz Generalse- \\ kretariat in Berlin und koordiniert \\ das Projekt "stark für Erfolg". \\ younisd@drk.de
}

\author{
Kinder und Jugendliche mit Migrationshintergrund haben \\ in Deutschland häufig immer noch schlechtere Bildungs- \\ und Berufschancen. Wie durch die Zusammenarbeit mit \\ Eltern und Partnern vor Ort die Bildungsverläufe von \\ Anfang an besser begleitet werden können, erkundet das \\ Projekt "stark für Erfolg" des Deutschen Roten Kreuzes.
}

Der Zusammenhang zwischen Herkunft und Bildungserfolg ist in Deutschland stark ausgeprägt. Dies hat in diesem Jahr der 14. Kinder- und Jugendbericht der Bundesregierung erneut gezeigt (BMFSFJ 2013, S. 243 ff.). Insbesondere an den Übergängen im Bildungssystem vollzieht sich die Weichenstellung, die vielfach über die Chancen von Mädchen und Jungen verschiedener sozialer und kultureller Herkunft entscheidet.

Diesem Thema widmet sich derzeit das Projekt »stark für Erfolg - Begleitung von Kind und Familie bei Bildungsübergängen« des Deutschen Roten Kreuzes. Schon seit einiger Zeit unternimmt der Verband mit seiner Strategie der interkulturellen Öffnung große Anstrengungen, um Menschen mit Migrationshintergrund sowohl innerhalb der eigenen Organisation als auch in den verschiedenen Aufgabenfeldern des Verbands die gleichen Chancen auf Teilhabe zu eröffnen.

Und Teilhabe beginnt mit Bildung: An 30 Standorten bundesweit werden daher seit April 2012 bestehende Strukturen der Familienbildung im Deutschen Roten Kreuz ausgebaut und Kooperationen mit Bildungsinstitutionen und $\mathrm{Mi}$ grantenorganisationen geschlossen, um Eltern bei Fragen rund um die Bildung ihrer Kinder zu beraten. Die beteiligten Einrichtungen öffnen sich damit auch einer stärkeren Beteiligung der Eltern. Das Modellprojekt wird vom Bundesministerium für Familie, Senioren, Frauen und Jugend gefördert und läuft bis Ende 2014. Es wird wissenschaftlich begleitet und evaluiert, um Erkenntnisse über
Zugänge und Methoden der Bildungsbegleitung für Familien mit Migrationshintergrund zu gewinnen.

Das Projekt strebt eine partnerschaftliche Zusammenarbeit mit den Eltern an und adaptiert damit das Modell der Erziehungspartnerschaft an die Bildungsthematik. Neben der Kooperation mit den Eltern und Öffnung für ihre Mitwirkung sehen Erziehungs- und Bildungspartnerschaften auch eine kommunale oder lokale Vernetzung im Sinne von »Bildungslandschaften« oder »Präventions- und Bildungsketten « vor (Stange 2012, S. 12 ff.). Die konkrete Angebotsgestaltung richtet sich folgerichtig nach dem Bedarf und den Ressourcen an den jeweiligen Praxisstandorten, wobei sich die Arbeit im Gesamtprojekt konzeptionell an vier Zielen orientiert:

- Zugangswege verbessern: Das Projekt dient der Erprobung von Zugangswegen zur Bildungsbegleitung insbesondere für Familien mit Migrationshintergrund. Zu diesem Zweck arbeiten vor Ort unterschiedliche Akteure wie Kinder-, Jugend- und Familienhilfe, Integrationsagenturen, Schulen und Migrantenorganisationen zusammen. Durch die Einbindung von Angeboten der Familienbildung können Eltern früh erreicht und von Vertrauenspersonen für das Thema sensibilisiert werden. Gleichzeitig werden durch Gehstrukturen auch Zielgruppen angesprochen, die bislang in der Familienbildung unterrepräsentiert sind (vgl. BMFSFJ 2013, S. 247). 
- Elternkompetenz stärken: Ziel des Projekts ist die Stärkung der Elternkompetenz in Bezug auf die Bildungsverläufe ihrer Kinder. Im Unterschied zu Programmen zur Förderung der allgemeinen Erziehungskompetenz findet eine inhaltliche Fokussierung auf Fragen der Bildung statt. Dabei wird ein erweiterter Bildungsbegriff zugrunde gelegt, der formale, non-formale und informelle Bildung gleichwertig berücksichtigt. Die Spanne der Themen im Projekt reicht von der frühkindlichen Förderung über eine Sensibilisierung für die Bedeutung von Bildung im Familienalltag bis hin zu den konkreten Bildungsentscheidungen. Dabei werden alle Bildungsübergänge betrachtet: der Übergang in die Kindertagesstätte, Grundschule, weiterführende Schule und in die Ausbildung.

- Fachkräfte qualifizieren: Die Mitarbeiterinnen und Mitarbeiter in den Standorten, viele von ihnen selbst mit Migrationshintergrund, können sich im Rahmen des Projekts zu Fachkräften der Eltern- und Bildungsbegleitung weiterbilden. Auf diese Weise wird die Nachhaltigkeit der Arbeit über die Projektlaufzeit hinaus gesichert. Die Qualifizierung der Elternbegleiterinnen und Elternbegleiter wird in Kooperation mit der Bundesarbeitsgemeinschaft Familienbildung und Beratung durchgeführt.

- Ergebnisse evaluieren: Es gibt einen Mangel an evaluierten präventiven Programmen, die speziell auf die Be- dürfnisse von Familien mit Migrationshintergrund ausgerichtet sind. Mit der Evaluation des Projekts »stark für Erfolg « durch die Forschungsgruppe FGAT um Prof. Dr. Detlef Krüger von der Hochschule für Angewand- raussetzung dafür ist eine Atmosphäre des Willkommen-Seins und der Wertschätzung, in der nicht zwischen "uns « und »ihnen « differenziert und dadurch Abstand (und damit Ausgrenzung) geschaffen wird. Nur so werden zuge-

\section{"Wer Eltern erreichen will, sollte das Kind in den Mittelpunkt stellen"}

te Wissenschaften Hamburg soll ein Beitrag geleistet werden, um Erkenntnisse im Themenkomplex Migration und Bildungschancen zu gewinnen (vgl. Abb.)

\section{Interkulturelle und sozialräumliche Öffnung}

In der derzeitigen Phase des Projekts steht die Verbesserung der Zugänge für Familien mit Migrationshintergrund im Zentrum. Die bisherigen Erfahrungen zeigen, dass die Angebote der Bildungsbegleitung nur greifen, wenn Elternarbeit und eine bewusste interkulturelle Öffnung der beteiligten Bildungsinstitutionen und Einrichtungen der Kinder-, Jugend- und Familienhilfe Hand in Hand gehen.

Der Begriff der interkulturellen Öffnung wird hier im Sinne einer Barrierefreiheit gegenüber »Individuen, egal welche Unterschiede sie mitbringen oder ihnen zugeschrieben werden « gebraucht (Terkessidis 2010, S. 141). Vo-

\section{Evaluationsmodell im Projekt „Stark für Erfolg“}

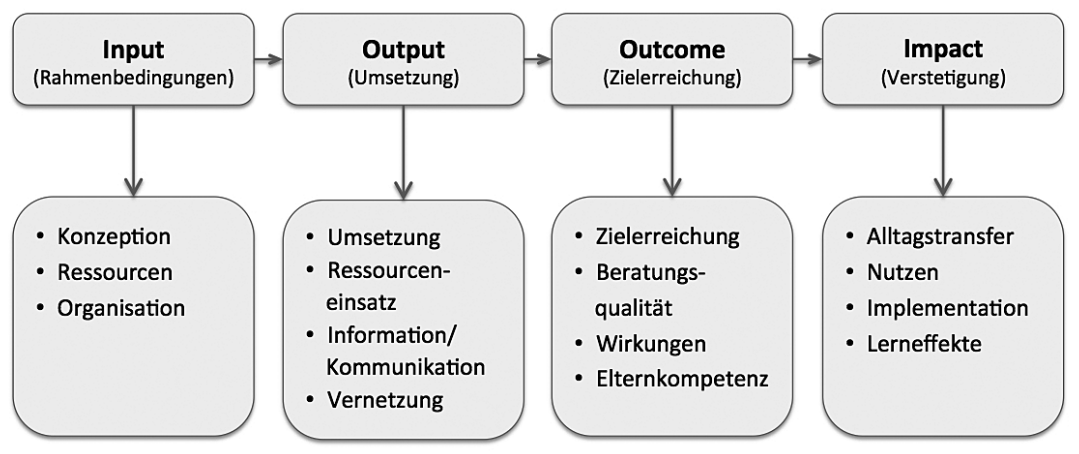

Das Projekt des Deutschen Roten Kreuzes "stark für Erfolg" wird durch die Forschungsgruppe FGAT von der Hochschule für Angewandte Wissenschaften Hamburg nach einem abgestuften Konzept umfassend evaluiert.

wanderte Eltern im deutschen Bildungssystem "ankommen" und ihre Kinder keine Nachteile erfahren. In einem Projekt, das bei der Elternkompetenz ansetzt, sollte es nicht so sehr darum gehen, Eltern diesem System »anzupassen ", als vielmehr sie im Umgang damit zu stärken und zu ermutigen, sich die Strukturen anzueignen.

In der Arbeit der Projektstandorte haben sich Faktoren herauskristallisiert, die die Beteiligung von Eltern mit Zuwanderungsgeschichte erleichtern. Einige Beispiele sind:

- Das Kind ins Zentrum stellen: Um die Eltern zu erreichen, sollte es um das Kind gehen. Angebote, welche primär die Beratung von sogenannten benachteiligten Eltern in den Vordergrund stellen, können defizitorientiert und stigmatisierend wahrgenommen werden. Das Interesse am Wohle der Kinder hingegen eint (fast) alle Eltern. Soziale und kulturelle Unterschiede treten dabei in den Hintergrund. Beispiel Offenbach: Die Elterngesprächskreise zu Bildungsfragen heißen bei der dortigen DRK-Familienbildung "Jedes Kind ist einzigartig «. Als Einstieg stellen alle Eltern, auch die Gruppenleiterin, die Vornamen ihrer Kinder vor. Sie erzählen, was ihr Kind einzigartig macht: Der Blick wird auf die Kompetenzen gerichtet.

- Nah bei den Menschen sein: Zu den zentralen Gelingensfaktoren gehören in diesem Projekt der niedrigschwellige Zugang und eine möglichst sozialraumbezogene Arbeitsweise: Man muss da hingehen, wo die Menschen sind, um sie zu erreichen. Die Angebote finden in Treffpunkten direkt in den Stadtteilen, in Kitas, Schulen und Moscheen statt. Auf diese Weise werden die Familien in ihrem unmittelbaren Umfeld, in ihrer Le- 
Wie »stark für Erfolg« wirkt

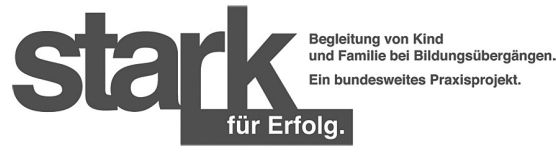

Um die Bildungschancen von Kindern und Jugendlichen mit Migrationshintergrund zu verbessern, initiiert das Projekt des Deutschen Roten Kreuzes "stark für Erfolg - Begleitung von Kind und Familie bei Bildungsübergängen " modellhaft an 30 Standorten bundesweit Bildungspartnerschaften mit den Eltern.

Ziel des Projekts ist die Stärkung der elterlichen Kompetenz zur Begleitung der Bildungsverläufe ihrer Kinder. Mädchen und Jungen verschiedener sozialer und kultureller Herkunft sollen dadurch ihre Talente erfolgreich entwickeln und zu eigenverantwortlichen und gemeinschaftsfähigen

benswelt angesprochen (vgl. zu Sozialraumorientierung u.a. Hinte/Treeß 2007). Die Integrationsagentur des Deutschen Roten Kreuzes in Hamm beispielsweise unterhält in zwei Wohnvierteln Treffpunkte, in denen Familien ein und aus gehen. Die Ansprechpartnerinnen, selbst Anwohnerinnen mit Migrationserfahrung, kommen leicht mit den Eltern ins Gespräch. Einem anderen Projektstandort, der mobilen Jugend- und Sozialarbeit Ellwangen des DRKKreisverbands Aalen, gelingt es über Streetwork und aufsuchende Arbeit auch marginalisierte Familien zu erreichen und die Bildungswege der Kinder und Jugendlichen über lange Zeit zu begleiten. Auch hier ist der persönliche Kontakt entscheidend.

- Begegnungsmöglichkeiten schaffen: Um Eltern zu aktivieren, sollten Gelegenheiten für Austausch und Mitgestaltung geschaffen werden. Viele der Standorte im Modellprojekt »stark für Erfolg « initiieren daher Elterncafés an Kindertagesstätten und Grundschulen. So auch das Projekt SIGNAL des DRK-Landesverbandes Saarland. Jeden Mittwoch früh können sich die Eltern, nachdem sie ihre Erstklässler zur Schule gebracht haben, im Elterncafé treffen und austauschen. Regelmäßig werden hier bildungsrelevante Themen vorgestellt und besprochen, beispielsweise wie die Kinder zuhause bei den Hausauf-
Persönlichkeiten heranwachsen. Durch die Zusammenarbeit unterschiedlicher Akteure wie Kinder-, Jugend- und Familienhilfe, Schulen und Migrantenorganisationen werden an den Projektstandorten neue Zugangswege und tragfähige Netzwerke entwickelt.

Bildung wird im Projekt in einem weiten Sinne verstanden und nicht auf Schulerfolg eingeengt

Das Projekt setzt auf Empowerment und auf die sozialräumliche und interkulturelle Öffnung der beteiligten Einrichtungen und Bildungsinstitutionen. In der Praxis hat sich gezeigt, dass es neben der Stärkung der Elternkompetenz zur familialen Förderung der Kinder und Beratung an den Bildungsübergängen wichtig ist, den Prozess der Selbstorganisation von Eltern zu unterstützen und Möglichkeiten der Partizipation zu schaffen. www.drk.de

gaben unterstützt werden können. Dadurch wird der - auch für Eltern nicht immer einfache - Übergang vom Kindergarten- zum Schulkind erleichtert, und die Eltern sind von Anfang an als Partner mit an Bord. Mit der Zeit bringen sie sich selbst immer mehr bei der Gestaltung des Elterncafés und anderer Aktivitäten in der Schule ein. Auf diese Weise werden anfängliche Hemmschwellen und Missverständnisse auf beiden Seiten abgebaut. Die Öffnung von Kitas und Schulen für eine Mitwirkung der Eltern ist ein entscheidender Schritt hin zu einer Bildungspartnerschaft, bei der institutionelle und familiale Förderung ineinandergreifen.

- Selbsthilfe organisieren: Zu einer gelingenden Kooperation mit den Eltern gehört ein Umgang auf Augenhöhe, zum Beispiel indem die Angebote gemeinsam mit den Eltern entwickelt werden, anstatt sie mit einem vorgegebenen Konzept zu konfrontieren. Auf diese Weise kann eine hohe Bedarfsorientierung sichergestellt und die Asymmetrie im Verhältnis zwischen Beratenden und Klienten abgebaut werden. Die Eltern werden als Experten für ihr Kind und als wichtige Ressource wahrgenommen. Durch den EmpowermentAnsatz im Projekt werden sie in die Lage versetzt, ihre Interessen selbst zu vertreten. Geradezu modellhaft ist dieses Prinzip im Projekt JeKaMi
(»Jeder kann mitmachen«) der DRKKinder- und Jugendhilfe Hamburg verwirklicht: Eltern, in der Regel Mütter, aus verschiedenen Kulturkreisen werden zu Gruppenleiterinnen für Elterncafés und Elternkurse weitergebildet. Diese Multiplikatorinnen, die sich selbst als »Motivatorinnen « begreifen, stärken die Eltern nicht nur in ihrer Kompetenz, sondern unterstützen sie auch im Prozess der Selbstorganisation. An elf Standorten in Hamburg gibt es solche Elterngruppen, die bei Bedarf auch in Englisch, Farsi, Türkisch und Russisch geleitet werden.

- Kompetenzen bündeln: Wie die Beispiele zeigen, sind die Angebote im bundesweiten Netzwerk »stark für Erfolg « an unterschiedliche Einrichtungen angebunden. Die Vielfalt der Standorte, die sich in den Netzwerken vor Ort fortsetzt, ermöglicht einen äußerst fruchtbaren Erfahrungsaustausch. So bringt die Familienbildung ihr Know-how im Bereich der Elternarbeit ein und die Migrationsberatung ihre Expertise im Bereich des interkulturellen Dialogs. Die mobile Jugend- und Sozialarbeit und Elternbegleitungsprogramme wie HIPPY steuern ihre Erfahrungen mit aufsuchender Arbeit bei und Mehrgenerationenhäuser ihre Kompetenzen im Bereich des bürgerschaftlichen Engagements und der sozialraumorientierten Arbeit. Dieser Wissenstransfer macht »stark für Erfolg « zu einem lernenden Projekt.

\section{Literatur}

Bundesministerium für Familie, Senioren, Frauen und Jugend (BMFSFJ), 2013: 14. Kinderund Jugendbericht. Bericht über die Lebenssituation junger Menschen und die Leistungen der Kinder- und Jugendhilfe in Deutschland. Berlin. Hinte, Wolfgang; Treeß, Helga (2007): Sozialraumorientierung in der Jugendhilfe. Weinheim und München.

Stange, Waldemar; Krüger, Rolf; Henschel, Angelika; Schmitt, Christof (Hg.), 2012: Erziehungs- und Bildungspartnerschaften. Grundlagen und Strukturen von Elternarbeit. Wiesbaden.

Terkessidis, Mark, 2010: Interkultur. Berlin. 